Gregory Stephanopoulos and his colleagues at the Massachusetts Institute of Technology in Cambridge rewired key metabolic pathways and tweaked the structure and expression of certain enzymes in the yeast Yarrowia lipolytica. This allowed the organism to convert low-value carbon compounds into fatty molecules similar to transport fuels and other chemicals that are used in various industries.

Some diesel-like fuels were produced at higher concentrations than similar approaches have achieved, taking the yeast 'refineries' a step closer to cost-efficient industrial applications, the authors say.

Proc. Natl Acad. Sci. USA http:// doi.org/bqn8 (2016)

\section{QUANTUM PHYSICS}

\section{City-wide} \section{teleportation}

Two groups have

demonstrated quantum teleportation - the remote exchange of quantum states - across cities using ordinary fibre-optic links.

In quantum teleportation, pairs of particles - typically photons - are created that share a common quantum state. Each particle is sent to a different location, and manipulating one affects the measurable properties of the other, so enabling the transfer of information. The teams, one led by Jian-Wei Pan at the University of Science and Technology of China in Shanghai and the other by Wolfgang Tittel at the University of Calgary in Canada, demonstrated data transfer between devices that were several kilometres apart in Hefei, China, and Calgary, respectively. Although such a feat had been achieved before with visiblelight photons, the current work used infrared photons, which are compatible with existing communications networks.

Teleportation could enable future quantum computers to exchange data.

Nature Photon. http://dx.doi. org/10.1038/nphoton.2016.179; http://dx.doi.org/10.1038/ nphoton.2016.180 (2016)

\section{NEUROSCIENCE \\ Cone cells see white too}

Light-sensing cells in the eye that have long been associated with colour vision could also be involved in non-colour vision.

The retina is lined with colour-sensing cone cells, and with rod cells, which are responsible for night vision. Ramkumar Sabesan at the University of California, Berkeley, and his colleagues used light to stimulate individual cone cells in two male volunteers and asked them to report the colour they saw. The team found that one group of cones was involved in sensing red and green, whereas another, larger group allowed the participants to see white.

The findings may reflect an evolutionary trade-off that favoured high-resolution noncolour vision over the ability to see fine-grained colour.

Sci. Adv. 2, e1600797 (2016)

\section{EVOLUTION}

\section{How snakes lost venom genes}

The ancestors of rattlesnakes had - and then lost - key genes involved in producing prey-paralysing venom.

The venom of North

American diamondback rattlesnakes (Crotalus atrox, pictured, and C. adamanteus)

targets the muscles and blood of victims, whereas the venom of the closely related Mojave rattlesnake (C. scutulatus) attacks the nervous system. To determine how these venoms evolved, a team led by Noah

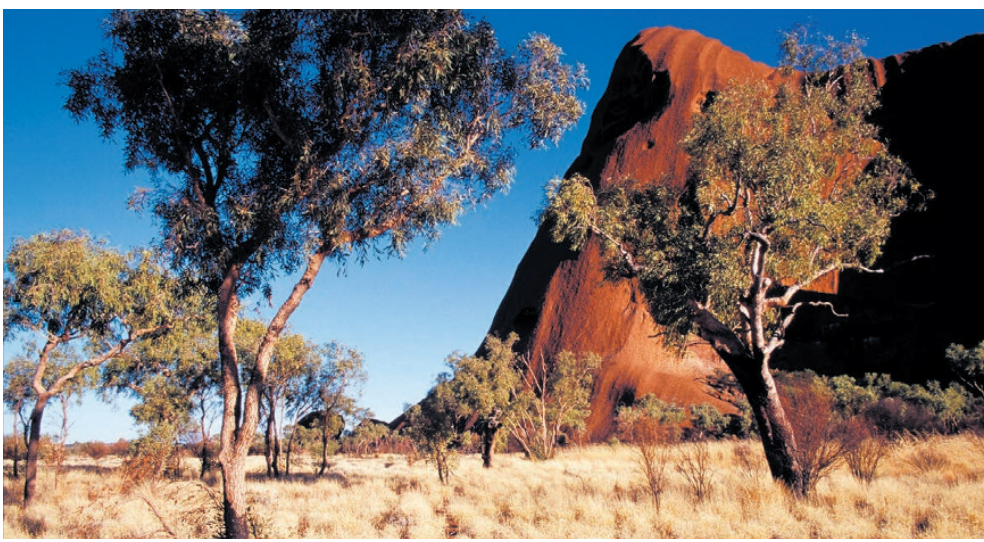

Dowell and Sean Carroll at the University of WisconsinMadison analysed the genomes of the three rattlesnakes and more distantly related snakes. Comparisons of venom genes suggest that the last common ancestor of rattlesnakes, which lived some 22 million years ago, had various toxin genes. The two diamondback lineages then independently lost the neurotoxin genes, and Mojave rattlers lost the genes to make muscle-attacking venom.

Shifting diets that included prey that were more resistant to a particular toxin may explain these losses, the authors say. Curr. Biol. http://doi.org/bqpn (2016)

\section{CLIMATE-CHANGE ECOLOGY}

\section{Australian tree range threatened}

Climate change could shrink the geographic range of most of Australia's eucalyptus species within the next 60 years.

Eucalypt trees (Eucalyptus, Corymbia and Angophora) are abundant in Australia (Eucalyptus terminalis and Corymbia opaca, pictured). Carlos González-Orozco, now at the Colombian Organisation for Agricultural Research in Meta, Bernd Gruber at the University of Canberra and their team used models to predict the distribution of some 650 tree species under various climate-change scenarios. They found that a $3^{\circ} \mathrm{C}$ rise over the next 60 years would, on average, reduce the climatically suitable habitat for $91 \%$ of the species by half. And some $90 \%$ of suitable habitats that host a high concentration of rare and evolutionarily old species could disappear or move south.

These losses could seriously threaten the diversity of the trees and their genetic ability to adapt to climate change. Nature Clim. Change http://dx. doi.org/10.1038/nclimate3126 (2016)

\section{ASTRONOMY}

\section{Universe much richer in galaxies}

The observable Universe is populated by between 1 trillion and 3 trillion galaxies, almost 10 times more than previously estimated.

A team led by Christopher Conselice at the University of Nottingham, UK, estimated this number using various telescope surveys that revealed evolving galaxy abundances since the time when the Universe was only about 600 million years old. The count included rare massive galaxies and smaller dwarf galaxies that can be impossible to see in the distant Universe with current telescopes.

The findings suggest that many more galaxies, especially faint and small ones, remain to be detected by projects such as the European Space Agency's upcoming Euclid mission.

Astrophys. J. in the press; Preprint at http://arxiv.org/ abs/1607.03909 (2016)

\section{NATURE.COM}

For the latest research published by Naturevisit:

www.nature.com/latestresearch 